\title{
Controlling Molecular Self-Assembly on an Insulating Surface by Rationally Designing an Efficient Anchor Functionality That Maintains Structural Flexibility
}

\author{
Christopher M. Hauke, ${ }^{\dagger,+}$ Ralf Bechstein, $^{\dagger}$ Markus Kittelmann, $^{\dagger}$ Christof Storz, $^{\S}$ Andreas F. M. Kilbinger, ${ }^{\S}$ \\ Philipp Rahe, ${ }^{\dagger, \perp}$ and Angelika Kühnle ${ }^{\dagger, *}$ \\ ${ }^{\dagger}$ Institute of Physical Chemistry, Department of Chemistry, Johannes Gutenberg-University Mainz, Duesbergweg 10-14, D-55099 Mainz, Germany, ${ }^{\ddagger}$ Graduate School \\ of Excellence Materials Science in Mainz, Johannes Gutenberg-University Mainz, Staudinger Weg 9, D-55128 Mainz, Germany, and ${ }^{\S}$ Department of Chemistry, \\ University of Fribourg, Chemin du Musée 9, CH-1700 Fribourg, Switzerland. 'P Present address: Department of Physics and Astronomy, The University of Utah, \\ 115 South 1400 East, Salt Lake City, Utah 84112-0830, United States.
}

\begin{abstract}
Molecular self-assembly on surfaces is dictated by the delicate balance between intermolecular and molecule-surface interactions. For many insulating surfaces, however, the molecule-surface interactions are weak and rather unspecific. Enhancing these interactions, on the other hand, often puts a severe limit on the achievable structural variety. To grasp the full potential of molecular self-assembly on these application-relevant substrates, therefore, requires strategies for anchoring the molecular building blocks toward the surface in a way that maintains flexibility in terms of intermolecular interaction

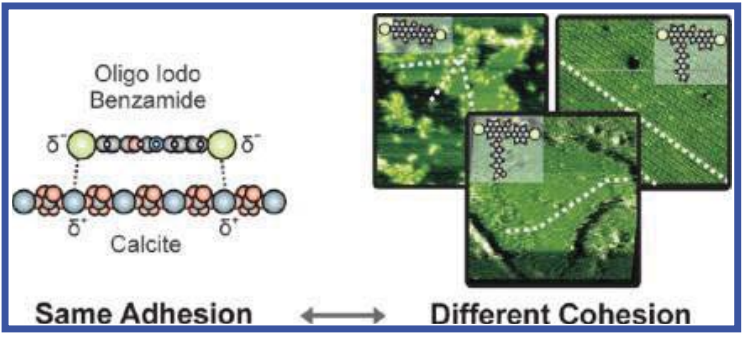
and relative molecule orientation. Here, we report the design of a site-specific anchor functionality that provides strong anchoring toward the surface, resulting in a well-defined adsorption position. At the same time, the anchor does not significantly interfere with the intermolecular interaction, ensuring structural flexibility. We demonstrate the success of this approach with three molecules from the class of shape-persistent oligo(p-benzamide)s adsorbed onto the calcite(10.4) surface. These molecules have the same aromatic backbone with iodine substituents, providing the same basic adsorption mechanism to the surface calcium cations. The backbone is equipped with different functional groups. These have a negligible influence on the molecular adsorption on the surface but significantly change the intermolecular interaction. We show that distinctly different molecular structures are obtained that wet the surface due to the strong linker while maintaining variability in the relative molecular orientation. With this study, we thus provide a versatile strategy for increasing the structural richness in molecular self-assembly on insulating substrates.
\end{abstract}

KEYWORDS: self-assembly $\cdot$ bulk insulator $\cdot$ substrate templating $\cdot$ NC-AFM $\cdot$ electrostatic anchoring

M olecular self-assembly is a powerful route for creating functional structures in a spontaneous and parallel fashion, that is, without the need to manipulate individual molecules one by one. ${ }^{1}$ On surfaces, self-assembly is controlled by the delicate balance between intermolecular and molecule-surface interactions, providing a highly effective key for tuning structure formation. In the past, an impressive range of molecular structures has been created, ${ }^{2}$ including perfect twodimensional overlayers, ${ }^{3}$ unidirectional rows, ${ }^{4,5}$ clusters, ${ }^{5,6}$ and porous networks ${ }^{7}$ with surface structures and surface reconstructions having significant influence on the molecular arrangements. ${ }^{8}$ These studies have, however, mostly been limited to metallic substrates as many classical surface-sensitive tools such as scanning tunneling microscopy or photoelectron spectroscopy are limited to conducting substrates. Besides this practical aspect of experimental accessibility, dielectric substrates pose another, more fundamental challenge for molecular self-assembly: most dielectric surfaces studied so far exhibit only very weak and
* Address correspondence to

kuehnle@uni-mainz.de. 
unspecific binding toward molecular building blocks. This weak interaction severely hampers the formation of substrate-templated, self-assembled structures on prototypical insulating surfaces held at room temperature. Instead, high molecular mobility, ${ }^{9}$ clustering at step edges, ${ }^{10}$ and molecular bulk crystal formation ${ }^{11}$ have been observed frequently. Thus, molecular selfassembly on insulating surfaces requires an increased molecule-surface interaction. Therefore, it is highly desirable to design molecules with specific anchor groups that provide a linker toward the surface. Several attempts have been made in this direction, most of them based on the electrostatic anchoring of molecules bearing a high dipole moment to an ionic crystal surface. ${ }^{9,12-14}$ When designing specific anchor groups, an important aspect needs to be considered: while strong binding toward the surface is desired for creating a stable wetting layer, the subtle balance between intermolecular and molecule-surface interactions is mandatory for maintaining structural flexibility. This fact can create conflicting interests as a strong binding and a well-defined adsorption position are usually incompatible with structural flexibility that is finetuned via the intermolecular interactions.

Here, we present a linking strategy based on the development of a versatile molecule from the class of shape-persistent oligo( $p$-benzamide)s that provides stable and site-specific anchoring of the molecular building block toward the surface of a bulk insulator, namely, calcite(10.4). Due to their inherently extended chain structure ${ }^{15}$ and their ability to form hydrogen bonds between chains, ${ }^{16-18}$ shape-persistent oligo( $p$ benzamide)s are promising candidates for use as supramolecular building blocks and the creation of molecular rods. ${ }^{18}$ The building block is specially designed to allow for electrostatic interaction of iodine atoms with the surface calcium ions, thus ensuring a strong and site-specific anchoring. At the same time, the iodine atoms do not significantly interfere with the intermolecular interaction that is encoded in the functional groups attached to the molecular core. Three oligo( $p$-benzamide)s are investigated that differ in their functionalization. While the adsorption position is found to be dictated by the iodine-calcium interaction, the relative orientation of the molecules with respect to each other is steered by the functionalization. This enables designing a variety of different molecular structures that are governed by the intermolecular interaction, despite the well-defined adsorption position. With this study, we demonstrate the successful linking of an organic building block to an insulating surface while at the same time maintaining structural flexibility.

\section{RESULTS AND DISCUSSION}

The molecules designed for this study are variations of 2-hydroxy-4-iodo- $N$-(4-iodophenyl)benzamide. Their structural models are shown in Figure $1 \mathrm{a}-\mathrm{c}$. The different

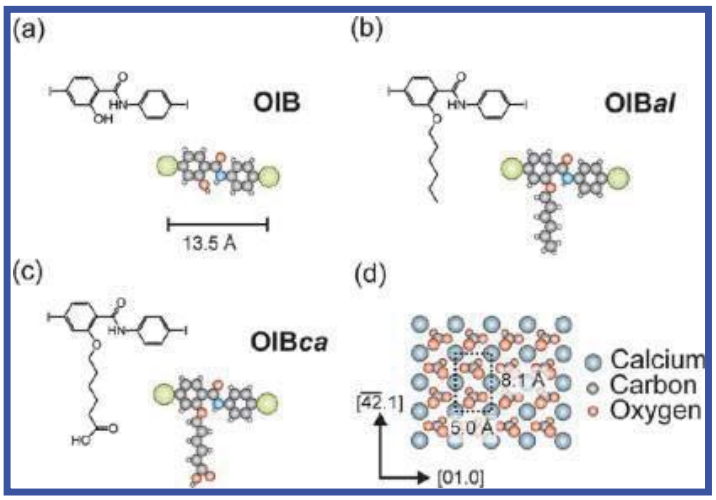

Figure 1. $(a-c)$ Skeleton formulas and ball models of the oligo( $p$-benzamide) molecules used in this study. (d) Model of the calcite(10.4) surface with a rectangular surface unit cell of $a \times b=8.1 \AA \times 5.0 \AA$. The carbonate groups are rotated such that one oxygen atom lies above, one in, and one below the plane spanned by the calcium ions.

molecules are named according to their functionalization. Due to the aromatic backbone of two iodobenzene units connected via an amide bond, the building block molecule (Figure 1a) is referred to as oligo iodo benzamide (OIB). The two other molecules are OIBal with an added hexyloxyl group (Figure $1 \mathrm{~b}$ ) and OIBca equipped with an additional carboxylic acid group at the end of the hexyloxyl chain (Figure 1c).

We investigate the self-assembly of these three molecules at room temperature (RT). The molecules were each deposited under ultrahigh vacuum conditions onto freshly prepared calcite(10.4) single-crystal surfaces. The surface unit cell of calcite(10.4) is rectangular with dimensions of $5 \AA \times 8.1 \AA$ (Figure 1d). Each unit cell contains two carbonate groups that are rotated with respect to the surface normal and with respect to each other. The comparatively high surface energy of $\gamma_{\mathrm{S}}=590 \mathrm{~mJ} / \mathrm{m}^{2}$ enables molecular wetting, making this insulating surface an ideal candidate for the study of molecular self-assembly. ${ }^{19}$

The imaging technique used for this study is noncontact atomic force microscopy (NC-AFM) in frequency modulation mode. All NC-AFM images presented are topography $(z)$ or detuning $(\Delta f)$ data where bright color corresponds to higher attractive tip-sample interaction.

Before we present the observed structures of the three different molecules, we discuss the expected interaction of the core building block with the calcite substrate. On the basis of the previous success of electrostatic anchoring, ${ }^{9,12-14}$ we make use of electronegative atoms that provide electrostatic attraction toward the surface calcium cations. The common core constituted by OIB is equipped with two iodine atoms at a center-to-center distance of $13.5 \AA$. It can be expected that the adsorption position is governed by a common motif, namely, that both iodine atoms are preferred to be centered directly above surface calcium ions, thereby maximizing the attractive moleculesubstrate interaction. When positioning one of the iodine atoms on top of a surface calcium cation, 
possible adsorption positions for the second iodine atom lie on a circle with a radius of $13.5 \AA$. The three Ca ions that lie closest to the circle, representing the three best molecular adsorption configurations in terms of iodine-calcium match, are presented in Figure 2. Table 1 summarizes the $\mathrm{Ca}-\mathrm{Ca}$ distances, angles, and deviations from the $\mathrm{I}-\mathrm{I}$ distance for these three best adsorption geometries. In the best two configurations (positions 1 and 2), the $1-I$ distance fits excellently to the distance of the underlying calcium ions with deviations smaller than 5\% (0.4 and $0.6 \AA$ ) while the third best choice (position 3) is already significantly less favorable with a deviation of $\sim 10 \%$ (1.3 A). Note that for each adsorption configuration there is an equivalent mirrored configuration with the mirror axis along ["ㄹ.1].

This purely electrostatic picture clearly favors two adsorption configurations (positions 1 and 2 in Figure 2), and we will demonstrate that indeed only these adsorption positions are adopted by the molecules in our study, justifying the simple electrostatic approach taken here.

As OIB, OIBal, and OIBca incorporate the same iodobenzene backbone, the molecular adhesion to the surface is expected to be very similar for all investigated molecules. The different functional groups contribute only marginally here, as will be confirmed later by our NC-AFM data. The functional groups will, however, influence the cohesion of the molecules on the surface as will be discussed individually for each molecule in the following sections.

OIB. Figure 3a shows the situation after depositing OIB onto the calcite surface at RT. We observe the formation of ordered, single-layered islands with an apparent height of about $3 \AA$. Considering the molecular dimensions, this height indicates that the molecules adsorb in a flat-lying manner on the surface. Following individual islands over several scan frames, we notice that their outline is changing with time, showing that the molecules at the edges of the islands are mobile at RT. Note that this observation is made even with very gentle tip-sample interaction strength. Apart from islands, we also observe mobile molecular species which are only visible as streaks in the island-free areas on the surface. Apparently, well-ordered islands and mobile molecules that attach at and detach from island edges coexist at RT. However, apart from cluster decorations on the island edges, as shown in Figure 3b, we never observe any second-layer growth. The absence of a second layer indicates the formation of a stable molecular wetting layer on the surface and sufficiently strong adhesion energy.

High-resolution NC-AFM imaging of single islands as depicted in Figure $3 \mathrm{~b}$ reveals that the islands consist of bright rows that are arranged in two domains I and II. Upon closer investigation, we find that the two domains constitute mirror images of each other, each enclosing an angle of $50^{\circ}$ with the substrate [ $\overline{42.1]}$ direction, which acts as the mirror axis. Considering the fact that the molecules lie flat on the surface, we expect intermolecular hydrogen bond formation between the

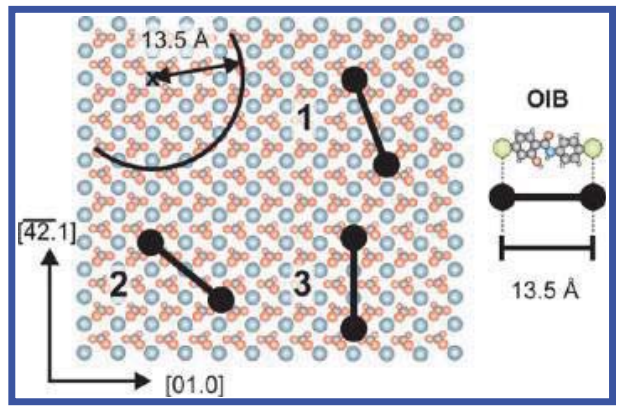

Figure 2. Determination of preferable adsorption positions for OIB on calcite(10.4). The black disks represent the iodine atoms with their covalent diameter. The three configurations that provide the best match of the distances between the molecule's iodine and the substrate's calcium atoms are drawn. For angles and distances, see Table 1.

TABLE 1. Structural Fit of the OIB Adsorption Positions from Figure 2

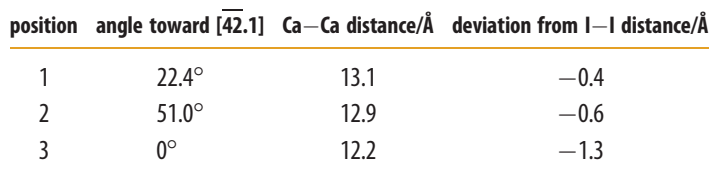

hydroxyl group and the carbonyl oxygen atom to be the driving force for the molecular arrangement on the surface. This interaction results in the formation of rows of molecules aligned side-by-side. The bright rows in the two domains have a repeat distance of approximately $16 \AA$, which is considerably smaller than the total length of the molecule of $17.5 \AA$. This finding indicates that the molecule's main axis forms an angle smaller than $90^{\circ}$ with respect to the row direction. We find an excellent side-by-side alignment of the molecules at an angle of about $72^{\circ}$ with respect to the row direction, as depicted in Figure $3 c$. From this molecular alignment and the known directions of the underlying calcite crystal, we determine the angle of the molecule with respect to the [ $\overline{42} .1]$ direction to about $22^{\circ}$, which is in excellent agreement with the angle of $22.4^{\circ}$ that is expected for the optimum adsorption position 1. Thus, we conclude that the simple picture of electrostatic interaction between the iodine atoms and the surface calcium cations is, indeed, correct for the OIB backbone.

Interestingly, the molecular islands can easily be destroyed during the scanning process. Depending on the interaction strength between tip and sample, islands can even be removed during the acquisition of a single image. In contrast to areas inside the island, where each molecule binds to two neighboring partners, the edge-located molecules have only one single binding partner. Thus, the disintegration process always starts at the edges of the molecular islands. The energy needed to separate one molecule from the island edge is most likely reduced at least to half the molecular binding strength inside the island. The relatively low cohesion of the molecules especially at the 

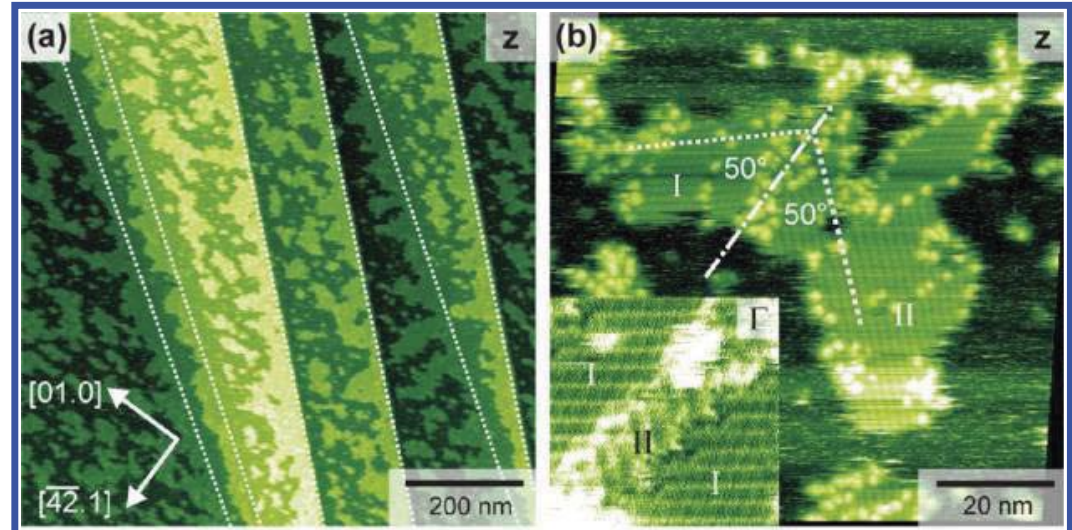

(c)

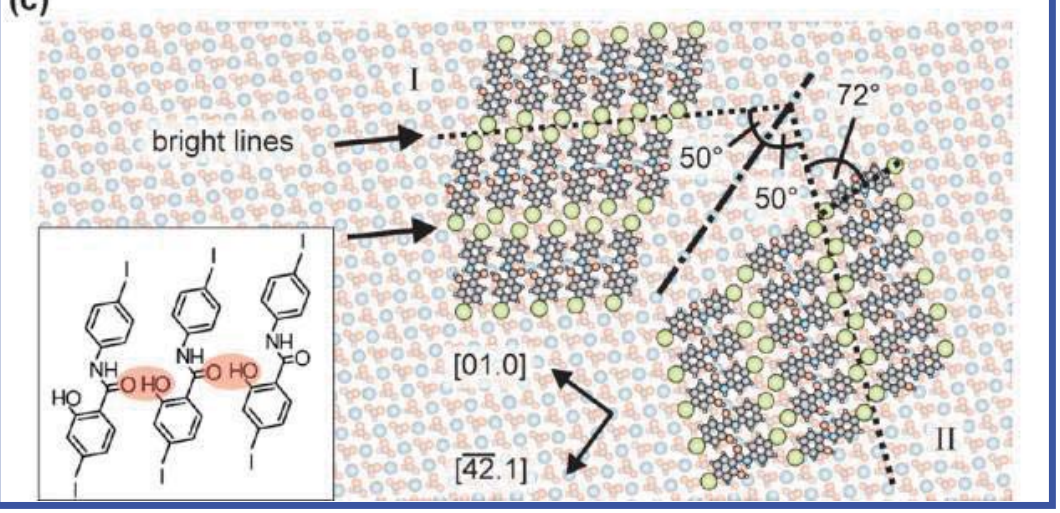

Figure 3. (a,b) NC-AFM topography $(z)$ data of OIB on calcite(10.4) at RT. The dashed lines in (a) indicate step edges of the underlying calcite substrate. Mirrored domains in (b) are labeled I and II. Their main orientations are indicated by dashed lines, and the mirror axis is given by the dash-dotted line. The image is corrected for linear drift. ${ }^{20}$ Inset of (b): Dissipation image revealing the row structure of domain I. The inset is magnified by a factor of 2 compared to (b). (c) Proposed molecular adsorption model. The hydrogen bond between two molecules is indicated by the shaded region in the inset.

island edges is, of course, highly undesirable when considering application-relevant conditions. While a successful anchoring of the molecules via the iodine atoms could be achieved, that is, the molecular adhesion is sufficiently strong, the intermolecular cohesion appears to be too weak. We, therefore, vary the intermolecular interaction by using functionalized OIB derivatives.

OIBal. We will now discuss how the structure formation is influenced by adding an alkyl chain to the OIB backbone, resulting in OIBal. Figure 4 a gives an overview image after depositing $\mathrm{OIBal}$ molecules onto the calcite(10.4) surface at RT. Very similar to OIB, islands with a height of $3 \AA$ are formed, again indicating a flatlying adsorption position. In contrast to the structures formed by OIB, however, we neither observe strike artifacts due to mobile molecular species in addition to island structures nor are the self-assembled islands decorated by clusters. This already indicates a stronger molecule-molecule interaction between OIBal molecules. A detailed analysis of images of the OIBal islands reveals the existence of two distinct domains I and II with a common orientation along the [01.0] direction of the underlying substrate (Figure 4b). High-resolution images reveal an internal network structure of these domains (Figure 4c). The repeat distance along the [01.0] direction is $15 \pm 1 \AA$ in both domains, which is exactly 3 times the repeat distance of the underlying calcite surface in this direction. This clearly emphasizes the template effect of the calcite substrate due to the anchoring of the iodine to the surface calcium cations. From the high-resolution image in Figure 4c, a model can be extracted that confirms the favorable adsorption position 1 of the molecular backbone (Figure $4 d$ ) exactly as in the case of OIB (see Figure 3c). The observed angle

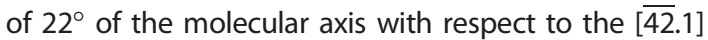
substrate direction constitutes a strong confirmation of adsorption configuration 1. Due to the additional alkyl chain, the OIBal molecules are forced to arrange in a less dense packing compared to OIB.

As a result, OIBal molecules form dimers connected via overlapping alkyl chains due to van der Waals interaction. Due to their very high flexibility, the interdigitated alkyl chains do not necessarily have to lie completely parallel to the surface but could protrude from the surface. The dimers arrange along the calcite [01.0] direction as shown in the model in Figure $4 \mathrm{~d}$. The back-to-back arrangement of the molecules is stabilized by the formation of two hydrogen bonds between the carbonyl oxygen atoms and adjacent hydrogen atoms in the neighboring molecule. Note that this is different from OIB, where the side-by-side alignment was stabilized by only one hydrogen bond. For geometric 


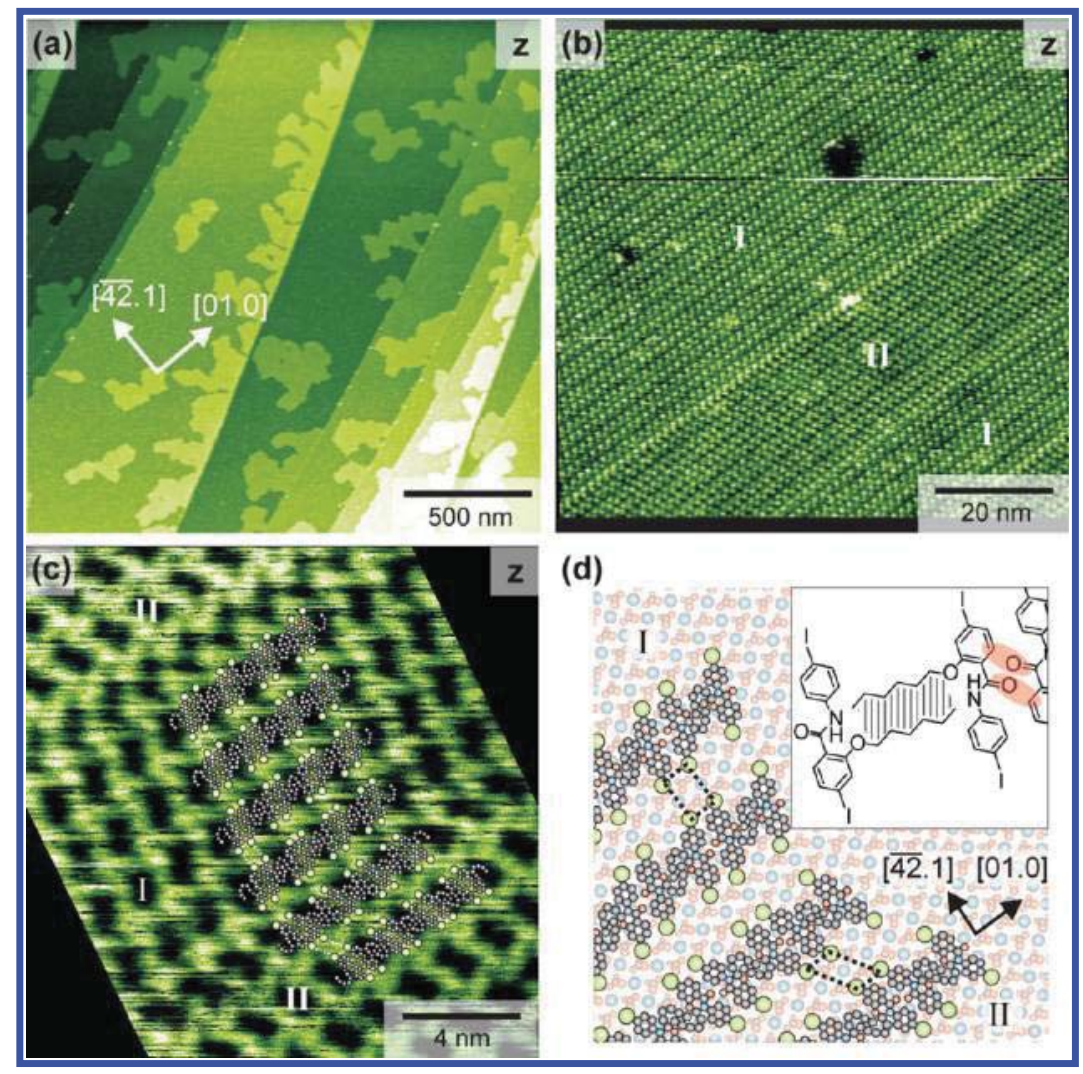

Figure 4. (a-c) NC-AFM topography (z) images of OIBal on calcite(10.4) at RT. Domains are labeled I and II. Images (b) and (c) are corrected for linear drift. ${ }^{20}$ (d) Proposed adsorption model. Hydrogen atoms are omitted for clarity. Translation of dimer rows along [01.0] causes two different arrangements of the row with respect to each other as indicated by the dashed connections of four iodine atoms in each domain. Inset: hydrogen bonds between two OlBal dimers are indicated by the shaded regions; the van der Waals interaction between the alkyl chains is indicated by the dashed area.

reasons, OIB molecules cannot be in a back-to-back configuration that allows for two hydrogen bonds between each pair of molecules because void regions would be created. OIBal molecules, in contrast, can arrange at a distance that allows for the formation of two hydrogen bonds in the back-to-back configuration since the alkyl chains serve as the necessary spacers that bridge these voids (see inset of Figure $4 d$ ). The required area per OIBal molecule is $152 \AA^{2}$ compared to $101 \AA^{2}$ for OIB. The dimer rows in both OIBal domains have a common orientation along [01.0] and a row distance of $20 \pm 1 \AA$. The adsorption position is mirror symmetric to

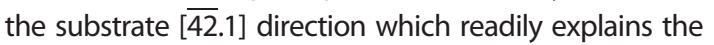
existence of the two domains. Additionally, two neighboring rows can be shifted with respect to each other, resulting in two possible arrangements, as shown in Figure $4 \mathrm{~d}$. This leads to frequent domain transitions and varying domain sizes.

The OIBal islands appear more stable than those formed by OIB molecules, as only slight rearrangements of the island edges are observed. This finding corroborates the above made statement of a stronger moleculemolecule interaction on the surface in the case of OIBal as compared to OIB. Even at tip-sample interactions larger than typical imaging conditions, it was not possible to deliberately destroy the OIBal islands by scanning. This is in sharp contrast to what was observed for OIB, where the low intermolecular cohesion is heavily interfering with the acquisition of high-resolution images. The higher cohesion of OIBal can be explained by the aforementioned interdigitation of the alkyl chains. It has been shown that the van der Waals interaction between two alkyl chains is on the order of $0.08 \mathrm{eV}$ per pair of interacting $\mathrm{CH}_{2}$ units, resulting in a total binding energy of about $0.5 \mathrm{eV}$ in the case of two interacting hexyl chains in an OIBal dimer. ${ }^{21,22}$ The interaction due to overlapping hexyl chains is considerably larger than the single hydrogen bond $(\sim 0.3 \mathrm{eV})$ between two OIB molecules, which is in excellent agreement with the experimental observation of less stable islands in the case of OIB.

Thus, by comparing the results of OIBal with those of OIB, we can conclude that while adhesion to the surface is very similar for both molecules, their cohesion differs significantly. Functionalization of OIB with an alkyl chain of sufficient length changed the type and strength of the molecular interaction, switching from a single hydrogen bond connecting two OIB molecules to van der Waals interaction and double hydrogen bonding for OIBal. The adjustment of the molecule design has a significant influence on the molecular structure formation, resulting in a distinct change in island morphology, density, and relative orientation of 


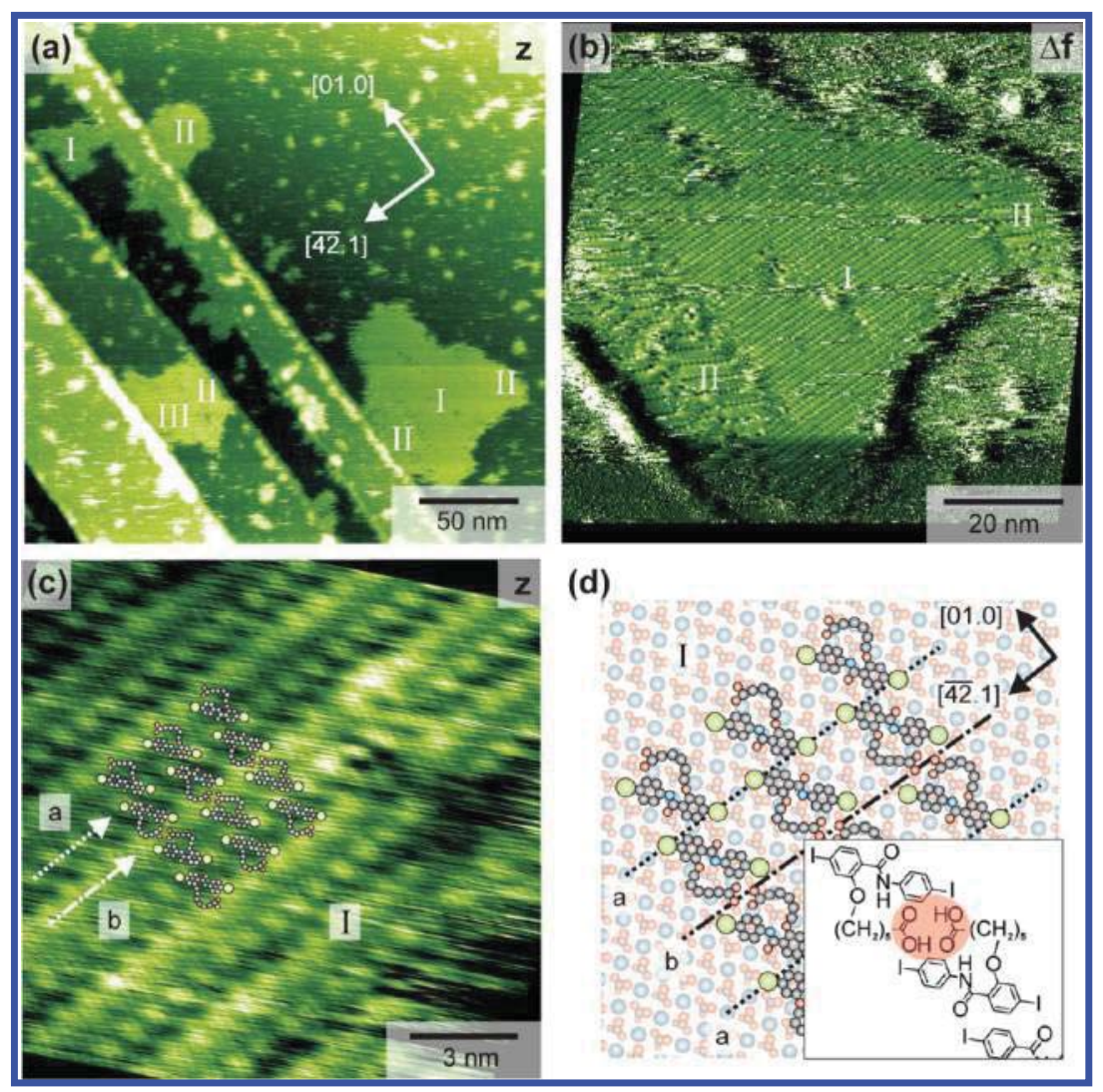

Figure 5. (a-c) NC-AFM topography $(z)$ and frequency shift $(\Delta f)$ images of OIBca on calcite(10.4) at RT. Three different domains are labeled I-III. (b) Detailed image revealing the internal structure of domains I and II. (c) Detailed image showing a domain I area. Images (b) and (c) are corrected for linear drift. The corrugated rows are marked with a dashed arrow and the blurry rows with a dash-dotted arrow. (d) Adsorption model for OIBca in position 2. Inset: carboxylic acid dimer is indicated by a shaded ellipse.

the molecules within the islands, despite the fact that the molecular adsorption position with respect to the underlying calcite substrate remained unchanged.

OIBca. Introducing a carboxylic acid group at the end of the OIBal alkyl chain yields the molecule OIBca. For this molecule, dimerization via the formation of hydrogen bonds between two carboxylic acid groups should be favorable. Since both $\mathrm{C}=\mathrm{O} \cdots \mathrm{H}$-O hydrogen bonds have a binding strength of about $0.3 \mathrm{eV}$, this dimer connection should be even stronger than the overlapping alkyl chains within an OlBal dimer ( $\sim 0.5 \mathrm{eV})$.

Figure 5 a shows the molecular structures that are formed after deposition of OIBca on the calcite(10.4) surface at RT. Ordered islands with a height of about $3 \AA$ form and coexist with mobile molecular species similar to the situation found after OIB deposition. In contrast to OIB, however, we do not observe decoration of the island edges.

High-resolution images reveal the existence of at least three different domains on the surface (Figure $5 a, b$ ). The molecular rows in domain I are oriented parallel to the substrate [42.1] direction, whereas the rows in domains II and III enclose an angle of \pm 29 and $\pm 34^{\circ}$ with that direction, respectively. In contrast to OIB, the different domains are not mirror images of each other. The coexistence of different domain structures indicates competing intermolecular interaction types of similar interaction strength. This is different from the welldefined configurations revealed for OIB and OIBal. In contrast to OIB and OIBal, OIBca presumably forms stable dimers that are connected at the end of two flexible alkyl chains, altogether resulting in much more flexible and space-consuming dimer units. This readily explains the structural complexity in the case of OIBca but at the same time precludes us from drawing a simple adsorption model for all configurations that are observed. We will, therefore, only exemplarily discuss a possible structural arrangement of OIBca molecules in domain I. Figure $5 \mathrm{C}$ shows a high-resolution image of domain I, revealing its inner structure. Two alternating row types, running parallel to the $[\overline{42} .1]$ direction are observed within the domain. Every second row has a clearly discernible corrugation with a periodicity of $7.8 \pm 0.5 \AA$ along [ [42.1], precisely matching the calcite repeat distance in that direction (a-type row). In contrast, every other row appears somewhat blurry (b-type row). A model for domain I is given in Figure $5 \mathrm{~d}$. The corrugation in the type-a rows is readily reproduced by OIBca dimers, and the equidistant spots can be assigned to the molecule's iodine atoms occupying adsorption sites above calcium cations. While the molecular backbone is firmly anchored to the surface, the alkyl chains connecting the dimers are 
still flexible enough, likely leading to the blurry b-type rows. The molecular orientation on the substrate matches that of the favorable adsorption position 2 presented in Figure 2. Thus, domain I confirms the favorable adsorption motif that was found for OIB and OIBal.

The images obtained for OIBca demonstrate that adding a flexible intermolecular linker results in yet another molecular arrangement. In good agreement with the flexibility of the linker, coexisting equivalent structures are revealed that are governed by the intermolecular cohesion, while the adhesion mechanism to the surface is unaffected by the change in functionalization.

The existence of the island structures of the three OIB derivatives readily demonstrates the subtle balance between the diffusion barrier and the intermolecular interaction: If the diffusion barrier would dominate, functionalization of the molecule would have a marginal effect on the resulting structure. If the intermolecular interactions would dominate, substrate templating would be negligible. Thus, the structural variety observed here in conjunction with the well-defined, substrate-dictated adsorption positions provides clear evidence that the balance between the diffusion barrier and the intermolecular interaction is, indeed, subtle.

\section{CONCLUSIONS}

We demonstrate the systematic variation of molecular cohesion for a set of molecules with identical adhesion to an insulating substrate. To achieve this, we engineered the functionalization of shape-persistent oligo( $p$-benzamide)s by introducing different functional groups and investigating their effect on the structural formation on the insulating calcite(10.4) surface. The molecular core was designed to provide site-specific electrostatic anchoring toward the surface, while at the same time maintaining the flexibility to fine-tune the resulting structure by adjusting the intermolecular cohesion energy. The success of this strategy is based on a clear separation of the molecule-substrate interaction from the molecule-molecule interaction. We compare three rationally designed molecules based on the same molecular building block. The molecules all adopt a well-defined adsorption position that is governed by the same iodine-calcium interaction motif, indicating the strength of the iodine anchor. Despite the identical adsorption mechanism, distinctly different molecular structures could be achieved by changing the molecule functionalization. The different functionalizations encode a change in the orientation of the molecules with respect to each other and, thus, result in entirely different self-assembled structures. Our results show that sufficient molecule-surface anchoring can be achieved without restricting the structural flexibility that is needed for the design of complex molecular systems.

\section{METHODS}

Substrate. The calcite crystals were purchased from Korth Kristalle GmbH (Altenholz, Germany) and were cut to a rectangular cross section of $2 \times 4 \mathrm{~mm}^{2}$. They were degassed in situ at $550 \mathrm{~K}$ for $2 \mathrm{~h}$ to remove contaminants. For each experiment, the crystal was freshly cleaved ${ }^{23}$ in situ and annealed at $500 \mathrm{~K}$ for $1 \mathrm{~h}$ to remove surface charges.

Molecules. Synthesis procedures for OIB, OIBal, and OIBca as well as NMR and UPLC-MS data can be found in the Supporting Information. Using a quartz crystal microbalance setup, we verified that all molecules were suitable for sublimation, while at the same time determining their respective sublimation enthalpies $\Delta H_{\text {sub }}$. The obtained values were $\Delta H_{\text {sub }}=1.48 \pm$ $0.08 \mathrm{eV}$ for OIB, $\Delta H_{\text {sub }}=1.68 \pm 0.08 \mathrm{eV}$ for OIBal, and $\Delta H_{\text {sub }}=$ $1.21 \pm 0.01 \mathrm{eV}$ for OlBca. All values were very close, which can easily be explained by their common aromatic backbone. The deviations were caused by the different molecular functionalization, demonstrating the different interaction strength in the bulk phase.

All molecules were deposited onto the surface under ultrahigh vacuum conditions by sublimation from a home-built Knudsen cell. The maximum chosen sublimation temperatures were $T_{\text {sub }}=421 \mathrm{~K}$ for OIB, $T_{\text {sub }}=353 \mathrm{~K}$ for OIBal, and $T_{\text {sub }}=398 \mathrm{~K}$ for OIBca. During the deposition process, the substrate was kept at RT. The samples were transferred into the AFM situated in the same ultrahigh vacuum system immediately after deposition.

AFM Setup. Experiments were performed at RT in an ultrahigh vacuum system at a base pressure lower than $1 \times 10^{-10} \mathrm{mbar}$. The system was equipped with an atomic force microscope (VT AFM XA from Omicron, Taunusstein, Germany) using a phase-locked loop detector and amplitude controller (easyPLL Plus from Nanosurf, Liestal, Switzerland) for signal demodulation and oscillation excitation. We used n-doped silicon cantilevers
(Nanosensors, Neuchâtel, Switzerland) with resonance frequencies of about $300 \mathrm{kHz}$ (type PPP-NCH), excited to oscillation with amplitudes of about $10 \mathrm{~nm}$.

Prior to their use, cantilevers were $\mathrm{Ar}^{+}$sputtered at $2 \mathrm{keV}$ for $5 \mathrm{~min}$ to remove contaminants and the oxide layer.

Depending on the feedback loop settings, either frequency shift $(\Delta f)$ or topography $(z)$ images were obtained. The image type is given in the upper right corner in each image. The $\Delta f$ images are displayed such that bright corresponds to high attractive interaction while dark corresponds to less attractive or even repulsive interaction.

Conflict of Interest: The authors declare no competing financial interest.

Acknowledgment. Financial support from the German Research Foundation (DFG) through the SFB 625 Grant TP B17 is gratefully acknowledged. C.M.H. is a recipient of a fellowship through the Excellence Initiative (DFG/GSC 266). P.R. gratefully acknowledges financial support from the Alexander von Humboldt Foundation.

Supporting Information Available: Synthesis procedures for OIB, OIBal, and OIBca as well as NMR and UPLC-MS data.

\section{REFERENCES AND NOTES}

1. Whitesides, G. M.; Mathias, J. P.; Seto, C. T. Molecular SelfAssembly and Nanochemistry: A Chemical Strategy for the Synthesis of Nanostructures. Science 1991, 254, 13121319.

2. Barth, J. V. Molecular Architectonic on Metal Surfaces. Annu. Rev. Phys. Chem. 2007, 58, 375-407. 
3. V. Oison, V.; Koudia, M.; Abel, M.; Porte, L. Influence of Stress on Hydrogen-Bond Formation in a Halogenated Phthalocyanine Network. Phys. Rev. B 2007, 75, 035428-1035428-6.

4. Kühnle, A.; Molina, L. M.; Linderoth, T. R.; Hammer, B.; Besenbacher, F. Growth of Unidirectional Molecular Rows of Cysteine on $\mathrm{Au}(110)-(1 \times 2)$ Driven by AdsorbateInduced Surface Rearrangements. Phys. Rev. Lett. 2004, 93, 086101-1-086101-4.

5. Böhringer, M.; Morgenstern, K.; Schneider, W.-D.; Berndt R.; Mauri, F.; Vita, A. D.; Car, R. Two-Dimensional SelfAssembly of Supramolecular Clusters and Chains. Phys. Rev. Lett. 1999, 83, 324-327.

6. Kühnle, A.; Linderoth, T. R.; Besenbacher, F. Self-Assembly of Monodispersed, Chiral Nanoclusters of Cysteine on the $\mathrm{Au}(110)-(1 \times 2)$ Surface. J. Am. Chem. Soc. 2003, 125, 14680-14681.

7. Pawin, G.; Wong, K. L.; Kwon, K. Y.; Bartels, L. A Homomolecular Porous Network at a Cu(111). Surf. Sci. 2006, 313, 961-962.

8. Schreiber, F. Self-Assembled Monolayers: From "Simple" Model Systems to Biofunctionalized Interfaces. J. Phys.: Condens. Matter 2004, 16, R881-R900.

9. Such, B.; Trevethan, T.; Glatzel, T.; Kawai, S.; Zimmerli, L.; Meyer, E.; Shluger, A. L.; Amijs, C. H. M.; de Mendoza, P. Echavarren, A. M. Functionalized Truxenes: Adsorption and Diffusion of Single Molecules on the $\operatorname{KBr}(001)$ Surface. ACS Nano 2010, 4, 3429-3439.

10. Nony, L.; Bennewitz, R.; Pfeiffer, O.; Gnecco, E.; Baratoff, A.; Meyer, E.; Eguchi, T.; Gourdon, A.; Joachim, C. Cu-TBPP and PTCDA Molecules on Insulating Surfaces Studied by UltraHigh-Vacuum Non-contact AFM. Nanotechnology 2004 15, S91-S96.

11. Kunstmann, T.; Schlarb, A.; Fendrich, M.; Wagner, T.; Möller, R.; Hoffmann, R. Dynamic Force Microscopy Study of 3,4,9,10-Perylenetetracarboxylic Dianhydride on $\mathrm{KBr}(001)$. Phys. Rev. B 2005, 71, 121403-1-121403-4.

12. Schütte, J.; Bechstein, R.; Rohlfing, M.; Reichling, M.; Kühnle, A. Cooperative Mechanism for Anchoring Highly Polar Molecules at an lonic Surface. Phys. Rev. B 2009, 80, 205421-1-205421-5.

13. Hinaut, A.; Pujol, A.; Chaumeton, F.; Martrou, D.; Gourdon, A.; Gauthier, S. An NC-AFM and KPFM Study of the Adsorption of a Triphenylene Derivative on $\operatorname{KBr}(001)$. Beilstein J. Nanotechnol. 2012, 3, 221-229.

14. Maier, S.; Fendt, L.-A.; Zimmerli, L.; Glatzel, T.; Pfeiffer, O. Diederich, F.; Meyer, E. Nanoscale Engineering of Molec ular Porphyrin Wires on Insulating Surfaces. Small 2008, 4, 1115-1118.

15. Kwolek, S. L.; Morgan, P. W.; Schaefgen, J. R.; Gulrich, L. W. Synthesis, Anisotropic Solutions, and Fibers of Poly 1 ,4benzamide). Macromolecules 1977, 10, 1390-1396.

16. Seyler, H.; Kilbinger, A. F. M. Linear Organo-Soluble Poly( $p$ Benzamide). Macromolecules 2009, 42, 9141-9146.

17. Storz, C.; Schulze, M.; Kilbinger, A. F. M. Solubility and Aggregation Behavior of Dendronized Poly( $p$-Benzamides. Macromol. Rapid Commun. 2011, 32, 238-244.

18. Schulze, M.; Storz, C.; Kilbinger, A. F. M. Non-aggregating Poly(p-benzamide)s. Chimia 2012, 66, 258.

19. Rahe, P.; Lindner, R.; Kittelmann, M.; Nimmrich, M.; Kühnle, A. From Dewetting to Wetting Molecular Layers: C60 on $\mathrm{CaCO}_{3}(10-14)$ as a Case Study. Phys. Chem. Chem. Phys. 2012, 14, 6544-6548.

20. Rahe, P.; Bechstein, R.; Kühnle, A. Vertical and Lateral Drift Corrections of Scanning Probe Microscopy Images. J. Vac. Sci. Technol., B 2010, 28, C4E31-C34E38.

21. Salem, L. Attractive Forces between Long Saturated Chains at Short Distances. J. Chem. Phys. 1962, 37, 2100-2113.

22. Lavrich, D.; Wetterer, S. M.; Bernasek, S.; Scoles, G. Physisorption and Chemisorption of Alkanethiols and Alkyl Sulfides on Au(111). J. Phys. Chem. B 1998, 102, 3456-3465.

23. Tröger, L.; Schütte, J.; Ostendorf, F.; Kühnle, A.; Reichling, M. Concept for Support and Cleavage of Brittle Crystals. Rev. Sci. Instrum. 2009, 80, 063703-1-063703-4. 